\title{
Construindo Critérios de Julgamento em Avaliação: especialistas e satisfação dos usuários com a dispensação do tratamento do HIV/Aids
}

\author{
Building Evaluation Criteria: experts and user satisfaction with \\ pharmaceutical delivery services for HIV/Aids
}

Ângela Esher ${ }^{1}$

Elizabeth Moreira dos Santos ${ }^{2}$

Rachel Magarinos-Torres ${ }^{3}$

Thiago Botelho Azeredo ${ }^{1}$

\footnotetext{
${ }^{1}$ Núcleo de Assistência Farmacêutica, Escola Nacional de Saúde Pública, Fundação Oswaldo Cruz. Rua Leopoldo Bulhões 1480/sl. 630, Manguinhos. 21041-210 Rio de Janeiro RJ. aesher@ensp.fiocruz.br ${ }^{2}$ Laboratório de Avaliação de Situações Endêmicas Regionais (Laser) Departamento de Endemias Samuel Pessoa, Escola Nacional de Saúde Pública, Fundação Oswaldo Cruz ${ }^{3}$ Faculdade de Farmácia, Universidade Federal Fluminense
}

\begin{abstract}
A criterion is a typical tool in the evaluation field that can be defined as a standarddimension under which the object of an evaluation receives qualitative or quantitative judgments. During an evaluation, several rigorous methodological procedures are involved in development and application of suitable criteria to determine the value of the object being evaluated. This article presents a set of criteria to evaluate user satisfaction with HIV/Aids-related pharmaceutical delivery services. The criteria construction process involved consensus amongst different experts, from academia, NGOs, management, by means of the Delphi technique. This technique prescribes a series of formal steps towards a consensus of experts, based on pre-structured methodology and processes. The findings were systematically organized in a structure under which the resulting satisfaction criteria are hierarchically organized. Results suggest the importance of developing a methodological strategy in evaluation that involves participation of different actors and of enhancing knowledge on user satisfaction and pharmaceutical delivery services for HIV/Aids.
\end{abstract}

Key words User satisfaction, Delphi technique, HIV/Aids, Evaluation
Resumo Critério é uma ferramenta típica da avaliação e pode ser definido como uma dimensão-padrão em função da qual a realidade do objeto da avaliação receberá julgamento qualitativo elou quantitativo. Em uma avaliação, o procedimento de elaborar, esclarecer, negociar e aplicar critérios para determinar o valor (ou mérito) do objeto avaliado faz parte de um exercício metodológico que deve ser cuidadoso. Este artigo apresenta critérios de julgamento para avaliar a satisfação dos usuários com a dispensação dos medicamentos para o tratamento do HIV/Aids e seu processo de construção por meio de consenso entre diferentes atores sociais. Para estabelecer o consenso utilizou-se técnica de Delfos, uma técnica formal utilizada para consenso de especialistas com metodologia e processos estruturados. Neste estudo, foram considerados especialistas aqueles com experiência acadêmica e em gestão, bem como ativistas informados e comprometidos com a questão. Os achados foram sistematizados sob a forma de uma nova estrutura de satisfação, sob a qual os critérios construídos são articulados hierarquicamente. Buscou-se contribuir para o desenvolvimento de uma estratégia metodológica participativa em avaliação e ampliar o conhecimento sobre a satisfação dos usuários e a dispensação dos medicamentos para HIV/Aids.

Palavras-chaves Satisfação dos Usuários, Técnica Delfos, HIV/Aids, Avaliação 


\section{Introdução}

Em uma avaliação, o procedimento de elaborar, esclarecer, negociar e aplicar critérios para determinar o valor (ou mérito) do objeto avaliado faz parte de um exercício metodológico que deve ser cuidadoso e transparente. Segundo Alföldi ${ }^{1}$, critério é uma ferramenta típica da avaliação e pode ser definido como uma dimensão-padrão em função da qual a realidade do objeto da avaliação receberá julgamento qualitativo e/ou quantitativo. $\mathrm{O}$ cumprimento desta etapa do processo avaliativo tem um papel essencial e exige da equipe de avaliação habilidade para escolha de estratégias metodológicas adequadas que possibilitarão a tradução de mensurações e observações em juízos de valor ${ }^{2}$.

A proposta de se construir critérios para avaliar a satisfação do usuário não é tarefa simples tendo em vista a complexidade deste conceito e a sua difícil operacionalização, mas pode ser um campo fértil para o exercício teórico metodológico de distintas abordagens em avaliação. Neste estudo, que busca a construção de critérios para avaliar a satisfação dos usuários com a dispensação dos medicamentos para o tratamento do HIV/Aids, serão utilizados princípios da abordagem participativa, a qual se destaca por permitir não só a coleta, a análise e a disseminação de dados, mas também a aprendizagem deste processo e a potencializar as possíveis ações daí resultantes. A utilização desta abordagem pressupõe a colaboração de avaliadores e de não avaliadores, sendo uma cooperação entre pesquisadores, facilitadores e avaliadores profissionais com indivíduos ou comunidades diretamente interessadas em uma intervenção ou programa que esteja sendo avaliado ${ }^{3}$. Para sua execução, os métodos participativos como técnicas de consensos têm ganhado destaque, pois são processos com ingerência ativa do público que objetivam a tomada de decisão de forma democrática, uma vez que permitem a representação igualitária de diferentes setores (perspectivas, opiniões e valores). $\mathrm{Na}$ área da saúde, têm sido recomendados quando há a necessidade de se estudar um aspecto ético, social ou cultural, ou ainda tomar uma decisão política ou técnico-científica em uma área específica do conhecimento.

No exercício apresentado neste artigo, a capacidade avaliativa é compreendida como o processo de construção a partir dos sujeitos, muito mais que um movimento meramente técnico ${ }^{4}$. Ao selecionar, consultar, discutir e pactuar com especialistas envolvidos com a dispensação espera-se aprofundar a compreensão destes sujeitos sobre a intervenção estudada e a produção de critérios cognitivos para avaliar a satisfação com as atividades que a integram.

A dispensação é um importante componente da avaliação da assistência e compreende o último momento em que os profissionais da farmácia interagem diretamente com o cliente externo o paciente. Quando o foco da pesquisa está relacionado à assistência farmacêutica de quem vive com Aids, é necessário considerar a escassez de propostas que respondam às necessidades específicas de um programa construído de forma coletiva entre as três esferas de governo (federal, estadual e municipal), a sociedade civil e os usuários do sistema de saúde, e para uma população que ainda tem uma relação com os serviços de saúde repleta de estigma, discriminação e medo ${ }^{5}$.

No âmbito do SUS, o incentivo à participação comunitária e o fortalecimento do controle social têm como pressuposto uma concepção de usuário competente para avaliar, intervir e propor mudanças. A participação dos usuários em avaliações de programas ou serviços de saúde ganha importância em virtude de suas contribuições, pertinentes e realistas, para a melhoria do cuidado em saúde. Metodologias que utilizam a opinião dos usuários possibilitam ainda que princípios relativos aos direitos individuais e sociais, tais como aqueles expressos nos conceitos de humanização e de direitos do paciente sejam reafirmados ${ }^{6}$. Estudos de satisfação são utilizados como peça chave para melhor qualificar informações sobre necessidades, desejos e demandas dos usuários.

Este artigo, parte de uma tese de doutorado que objetiva contribuir para o desenvolvimento de modelos teóricos que subsidiem avaliações de satisfação dos usuários com a dispensação dos medicamentos para o tratamento do HIV/Aids no Brasil, apresenta critérios de julgamento para avaliar a satisfação e seu processo de construção por meio de consenso entre diferentes atores sociais. Em relação ao processo será abordada a estrutura inicial da satisfação dos usuários, a seleção dos participantes, os procedimentos e os instrumentos de trabalho. Os achados foram sistematizados sob a forma de uma nova estrutura de satisfação e serão, desta forma, apresentados e discutidos.

\section{Estrutura Inicial da Satisfação dos Usuários}

Em estudo prévio ${ }^{5}$ que objetivou a construção de modelos de avaliação para a satisfação dos usuários com a dispensação de medicamentos para o tratamento do HIV/Aids, foram formulados critérios (dimensões e subdimensões) utilizados pelos usuários para julgar sua satisfação. 
O foco na experiência corrente da dispensação e não no efeito da intervenção sobre a população-alvo determinou a escolha de três dimensões da categoria acesso $^{7}$, a disponibilidade, a oportunidade e a adequação para estruturar a satisfação. Estas dimensões definem teoricamente o domínio dos indicadores operacionais da intervenção, os quais permitem a apreensão da satisfação dos usuários em relação ao serviço prestado. Assim: Disponibilidade (availability) é definida pela relação entre o tipo e a quantidade de produtos e serviços necessários e o tipo e a quantidade de serviços oferecidos; Oportunidade (accessibility) como existência dos serviços no lugar e no momento em que tecnicamente é requerido pela situação de saúde dos usuários; e Adequação (accomodation) refere-se ao ajuste entre as características dos produtos e serviços e as expectativas e necessidades dos usuários (Figura 1).

O conceito de acesso a serviços de saúde aqui utilizado está relacionado ao grau de ajuste entre os clientes e o sistema, isto é, refere-se à relação entre o conjunto de obstáculos encontrados na busca da atenção (resistência) e a capacidade cor- respondente da população para superar tais obstáculos, isto é, poder de utilização $0^{7,8}$. Assim, acesso é entendido como uma categoria mediadora, capaz de abordar e medir a capacidade de produzir, oferecer serviços (ou produtos) e o consumo real de tais serviços, constituindo-se em domínio interessante para se mensurar a implementação do programa ${ }^{5}$.

As categorias acesso e satisfação representam conceitos fortemente imbricados ${ }^{9,10}$. Segundo Penchansky e Thomas ${ }^{7}$, os problemas com quaisquer das dimensões do acesso influenciam os clientes e o sistema de três formas mensuráveis: (1) utilização dos serviços; (2) modificação da satisfação do usuário; e (3) padrões de organização do cuidado como, por exemplo, menos tempo destinado a cada cliente, desvirtuamento do uso da emergência e outros. Dessa forma, acesso, conforme a proposição de Penchansky e Thomas $^{7}$, modificada por $\mathrm{WHO} / \mathrm{MSH}^{11}$ permite a formulação de estratégias para avaliar a intervenção operacionalmente e estabelecer vínculos teóricos necessários para se abordar a satisfação do usuário.

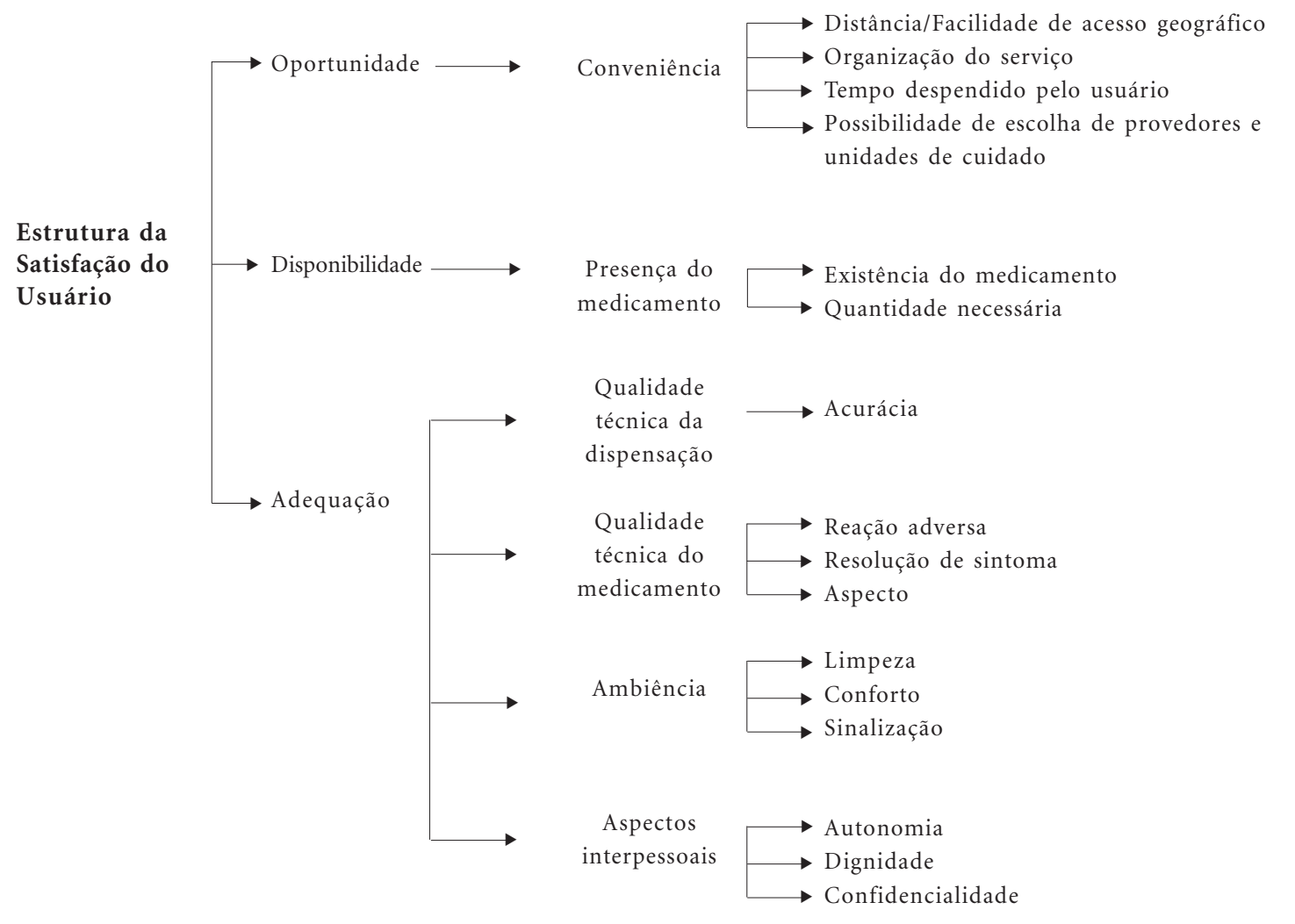

Figura 1. Estrutura inicial da satisfação dos usuários 
As dimensões eleitas para o modelo (Conveniência, Presença do Medicamento, Qualidade Técnica da Dispensação, Qualidade Técnica do Medicamento, Ambiência e Aspectos interpessoais) receberam subdimensões, selecionadas a partir da literatura que discute conceitos-chave e a aplicação de instrumentos utilizados para medir a satisfação do usuário/paciente ${ }^{6,12-15}$ (Figura 1).

Conveniência refere-se à distância/facilidade de acesso geográfico, organização do serviço, tempo despendido pelo usuário e possibilidade de escolha de unidades de saúde. Presença do medicamento refere-se à disponibilidade do medicamento na farmácia na quantidade prescrita para o usuário. Qualidade técnica da dispensação diz respeito à competência de provedores e à adesão aos componentes essenciais da dispensação. Qualidade técnica do medicamento está relacionada às características das embalagens e à apresentação dos medicamentos, à produção de reações adversas e à possível resolução de sintomas. Ambiência está relacionada com as características do local onde o cuidado é fornecido e deve garantir adequação das instalações às condições mínimas para um ambiente confortável (limpeza, sinalização, ventilação suficiente, lugar protegido de intempéries, água limpa para beber); Aspectos interpessoais estão relacionados à atitude dos dispensadores em relação aos usuários no momento do fornecimento dos medicamentos e inclui os componentes de autonomia, dignidade e confidencialidade.

\section{Aplicação de Técnica de Delfos como Forma de Ampliar a Participação dos Interessados no Processo Avaliativo}

Para estabelecer o consenso entre especialistas em relação aos critérios de julgamento para orientar a avaliação da satisfação dos usuários com a dispensação de medicamentos para o tratamento do HIV/Aids utilizou-se técnica de Delfos. Delfos (ou Delphi) é uma técnica formal utilizada para consenso de expertos (ou especialistas) com metodologia e processos estruturados. Tem como base a utilização do conhecimento, da experiência e da criatividade de um painel de especialistas, e como pressuposto a idéia de que o julgamento coletivo, organizado sistematicamente, é mais consistente que a opinião individual. Por esta técnica, estabelecer consenso significa adotar uma decisão de comum acordo entre duas ou mais partes. Experto (ou especialista) é aquele que possui experiência, habilidade ou determinados conhecimentos técnicos, científicos ou práticos sobre um tema específico. Nesta perspectiva ampliada, o conceito de especialista inclui des- de a experiência acadêmica até a vivência de pessoas bem informadas comprometidas com a questão estudada. Epstein ${ }^{16}$ discute de forma bastante consistente o papel dos ativistas e seus aliados na produção do conhecimento no campo da AIDS. A despeito de serem considerados "não especialistas", estes tem uma atuação que permite interferir, direcionar e participar da produção de diferentes modalidades de pesquisa assim como da construção de políticas públicas.

Segundo Wright e Giovinazzo ${ }^{17}$, a opção por Delfos e não por outras técnicas de consenso deve ser feita em função de peculiaridades do estudo, tais como: inexistência de dados históricos, necessidade de abordagem interdisciplinar e perspectivas de mudanças estruturais no setor. Se bem executada, esta técnica facilita o acesso a um grupo amplo e a uma população geograficamente dispersa de forma simultânea; e o intercâmbio de informações entre um número importante de pessoas; evita a coesão de grupo e impede que a autoridade de algum membro influencie outros; e permite que pessoas opinem sem se expor. No entanto, a seleção inadequada dos participantes, perguntas mal formuladas, problemas na estruturação da participação dos selecionados, método incorreto para sintetizar o juízo individual de cada participante e a influência de fatores externos ou de fontes de financiamento podem ser apontados com aspectos prejudiciais à sua aplicação.

A utilização de técnicas de consenso é apontada como útil para a validação da confiabilidade da informação ${ }^{18}$. Os autores argumentam que não se trata de uma validação "técnica, formal ou estatística” do que é medido pela informação, mas de uma análise subjetiva, oriunda dos profissionais ou especialistas sobre as situações de saúde que serão subsídios para monitoramento e avaliação. No campo da Avaliação de Programas de Saúde, seu uso tem sido crescente, provavelmente por representar uma iniciativa de fortalecimento da capacidade técnica em $\mathrm{M} \& \mathrm{~A}^{18}$.

O caráter inclusivo de Delfos possibilitou receber contribuições de especialistas, que utilizaram experiências reais vividas com o planejamento, a gestão, a dispensação ou o recebimento de medicamentos em diferentes áreas geográficas do país. Possibilitou ainda que cada participante expressasse suas idéias evitando situações constrangedoras que poderiam surgir no contato face a face $^{18}$. Além disso, representou baixo custo para a equipe do estudo.

\section{Seleção dos participantes}

Em 2005, o Núcleo de Assistência Farmacêutica (NAF/ENSP/FIOCRUZ) sob encomenda do 
Programa Nacional de DST/Aids (PN-DST/Aids), mais especificamente a Unidade de Assistência e Tratamento (UAT) e a Assessoria de Monitoramento e Avaliação (AM\&A), executou uma avaliação da dispensação de medicamentos para o tratamento das Pessoas Vivendo com HIV/Aids (PVHA). O estudo, conduzido em dez estados brasileiros indicados por amostra intencional, combinou critérios de magnitude da epidemia com a intenção de se obter um panorama geral da qualidade da dispensação de ARV e MIO no Brasil. Foram abordados dois eixos: a) aspectos relativos à estrutura disponível e aos processos pertinentes à produção dos efeitos esperados e; b) satisfação dos usuários com o processo de dispensação ${ }^{19}$.

Um dos efeitos da avaliação foi a constituição pelo PN-DST/Aids do Grupo de Trabalho (GT) em Assistência Farmacêutica, composto por cerca de 20 pessoas com diferentes inserções profissionais na área de medicamentos e de assistência farmacêutica e ativistas do movimento social de luta contra aids, para elaborar um documento com novos procedimentos a fim de melhorar esta intervenção.

Após a autorização concedida pelo PN-DST/ Aids, instância responsável pela organização do grupo, foram convidados todos os seus membros, além de duas farmacêuticas, responsáveis por dispensar medicamentos ARV na região Norte e na região Sudeste, uma especialista em avaliação de qualidade de serviço com inserção governamental e uma PVHA há cerca de 20 anos, com inserção acadêmica na área de comunicação e aids. Dos 24 especialistas convidados, 12 responderam ao convite, aceitando participar. Como o GT organizado pelo PN-DST/Aids já tinha encerrado suas tarefas, a participação de seus membros não comprometeu a independência das sugestões.

\section{Procedimentos}

\section{e Instrumentos de Trabalho}

Os 12 especialistas que concordaram em participar receberam por correio eletrônico, em mensagem individual, o primeiro instrumento de trabalho. O documento continha Termo de Consentimento Livre e Esclarecido (TCLE), texto introdutório sobre os objetivos do estudo e esclarecimento sobre o processo de construção de consenso utilizando Delfos, instruções para preenchimento e devolução do material. Em seguida, foi apresentada breve introdução sobre a definição de dispensação bem como das dimensões selecionadas para medir a satisfação do usuário/ paciente utilizadas no estudo. Solicitou-se contribuições sobre conteúdos que poderiam definir cada dimensão da satisfação, expressas de forma livre. Assim, o participante deveria construir itens para compor cada uma das dimensões mencionadas. Desta forma, por exemplo, o especialista deveria responder à seguinte questão: "Para o (a) senhor (a) Presença do Medicamento é ?"; e o exemplo de resposta possível: Presença do Medicamento é uma dimensão utilizada para o usuário avaliar sua satisfação quanto à disponibilidade do medicamento na farmácia na quantidade prescrita para ele. Inclui itens como "ter todos os medicamentos" e "não faltar medicamentos importantes". O texto trazia ainda a possibilidade de o participante acrescentar outros itens que considerasse importante, mesmo que não relacionados às dimensões mencionadas. O termo "item" é aqui utilizado como uma referência às contribuições dos especialistas (Quadro 1).

Nesta primeira etapa, o prazo para a devolução da resposta foi de 20 dias. Como nem todos responderam neste prazo foi enviada uma comunicação explicando a importância da participação dos especialistas e fornecida uma nova data. Após o segundo prazo, entre 10 e 15 dias depois, outros participantes responderam. Assim, do grupo inicial de doze que concordaram em participar do estudo, nove responderam.

O segundo instrumento de trabalho foi enviado 45 dias após o final do segundo prazo apenas para os nove que responderam ao primeiro. Nesta segunda rodada, os especialistas deveriam julgar os itens quanto à pertinência e à relevância. Foi estabelecido como item relevante aquele necessário, importante para medir a satisfação do usuário com a dimensão. Os itens poderiam receber Sim ou Não para a pertinência e pontuação de 0 (zero) a 2 (dois) para a relevância, onde 0 (zero) representava nada relevante, 1 (um) pouco relevante e 2 (dois) muito relevante (Quadro 1). Foi ressaltado para os participantes que não havia respostas corretas ou erradas.

$\mathrm{Na}$ análise da pertinência, para ser objeto de consenso, um item deveria ter recebido cinco ou mais respostas positivas ou negativas. No primeiro caso, considerou-se consenso quanto à pertinência destes itens; no caso de cinco ou mais respostas negativas, considerou-se consensual que o item não era pertinente para aquela dimensão.

Somente os itens considerados pertinentes foram avaliados quanto à relevância. O estabelecimento da pontuação permitiu determinar um valor de relevância para o item. A pontuação final de cada item foi calculada multiplicando o número total de respondentes que considerou o item pertinente pelo valor dos pontos recebidos. Isto resultou na pontuação final de cada componente. A 
Quadro 1. Estrutura dos instrumentos de trabalho utilizados na construção de critérios de julgamento em avaliação da satisfação dos usuários com a dispensação do tratamento do HIV/Aids.

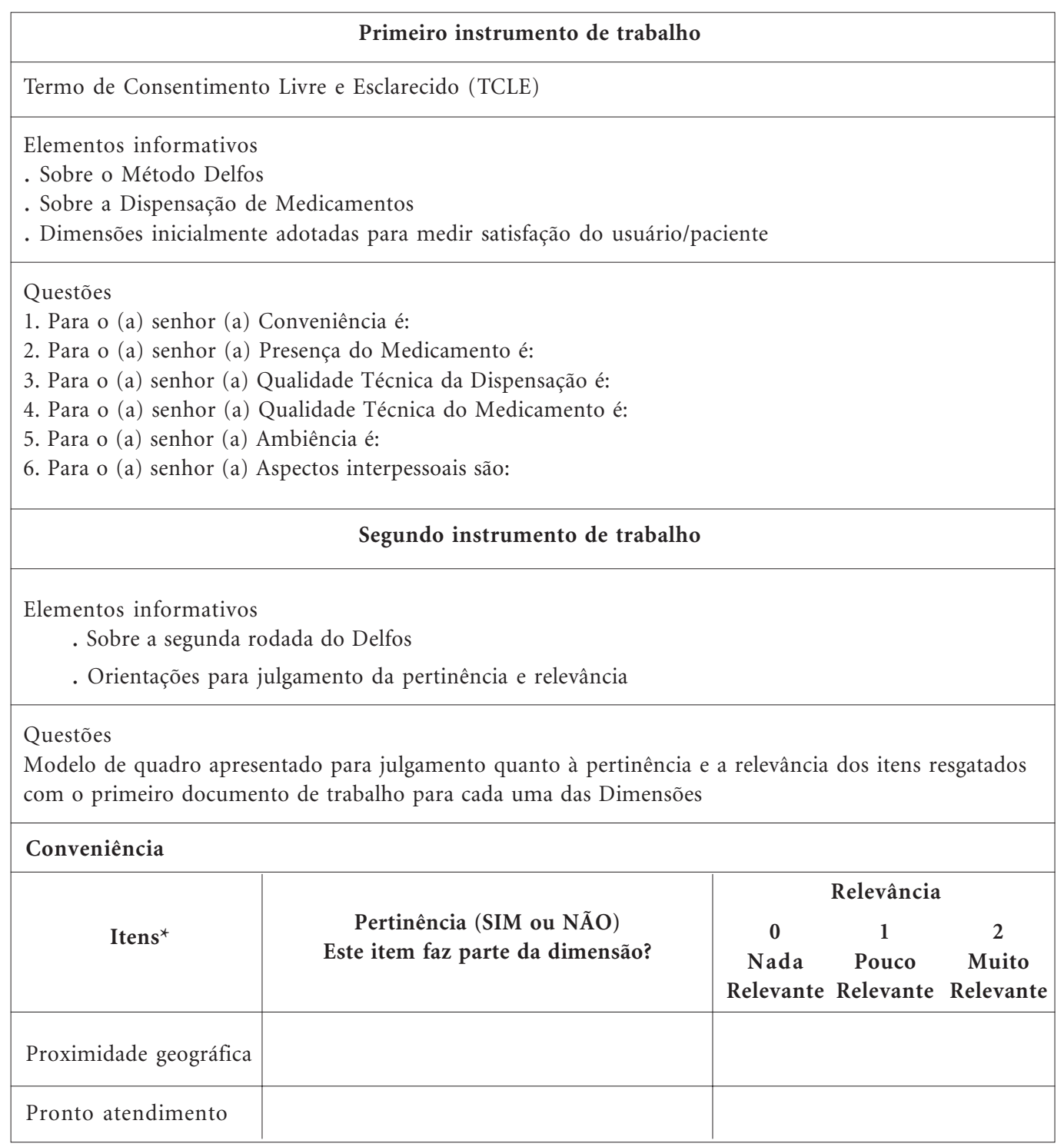

*No segundo instrumento de trabalho todos os itens resgatados com o primeiro documento foram apresentados para julgamento. Neste modelo apresentamos, como exemplo, uma das dimensões, dois itens e a forma como estava estruturada a questão.

pontuação variou de 0 a 14 . Foram considerados com alto grau de consenso quanto à relevância itens cuja pontuação foi igual ou superior a $80 \%$ do valor máximo alcançado. Itens sem consenso para pertinência não receberam esta pontuação.

Para a construção da nova estrutura de satisfação os itens foram analisados sendo, quando necessário, agregados ou decompostos, formando-se assim as novas subdimensões.

Este estudo está de acordo com as orientações presentes na Resolução CNS 196/96, foi submetido e aprovado pelo Comitê de Ética em Pes- quisa da Escola Nacional de Saúde Pública Sérgio Arouca.

\section{Estrutura da Satisfação após o Delfos}

A primeira rodada do Delfos identificou 108 itens para compor uma medida de satisfação dos usuários segundo os especialistas. Foram distribuídos da seguinte forma: 21 relacionados à dimensão Conveniência, 15 à dimensão Qualidade Técnica da Dispensação, 27 à dimensão Qualidade Técnica do Medicamento, 22 à dimensão Ambiência, 15 à dimensão Aspectos Interpessoais, 
e 8 à dimensão Presença do medicamento. Segundo estes dados, a dimensão Qualidade Técnica do Medicamento obteve maior quantidade de itens sugeridos que as demais, enquanto a dimensão Presença do Medicamento obteve a menor. Quanto maior o número de itens inicialmente listados menor o consenso inicial quanto aos critérios de julgamento.

A segunda rodada do Delfos enunciou 49 itens considerados como pertinentes pelo grupo de trabalho. Os itens se distribuíram por todas as dimensões da estrutura inicial da satisfação dos usuários. Nenhuma dimensão presente no modelo inicial ficou sem critério de julgamento. A disposição dos itens por dimensão aconteceu da seguinte forma: 9 relacionados à Conveniência, 11 à Qualidade Técnica da Dispensação, 13 à Qualidade Técnica do Medicamento, 7 com Presença do Medicamento, 4 com Ambiência e 5 com Aspectos Interpessoais (Tabela 1).

Confrontando a estrutura elaborada a partir dos itens gerados no Delfos (Figura 2), com a estrutura inicial da satisfação do usuário (Figura 1), todas as dimensões da satisfação aparecem caracterizadas por um número maior de subdimensões. As novas subdimensões ampliam o domínio das dimensões inicialmente construídas e por muitas vezes tornam tênues os limites entre as dimensões. Isto pode ser observado quando uma mesma subdimensão, como por exemplo, a orientação, aparece compondo diferentes dimensões (conveniência, qualidade técnica da dispensação e qualidade técnica do medicamento). Disponibilidade de medicamentos foi outra subdimensão que surgiu em mais de uma dimensão (conveniência e presença do medicamento).

A dimensão Conveniência recebeu subdimensões que ampliaram o conteúdo inicialmente elaborado. No modelo inicial, o foco estava na "conveniência da unidade dispensadora”, isto é, fazendo referência aos aspectos relacionados à organização e à localização do serviço para atender as necessidades dos usuários. Com o trabalho dos especialistas foram incluídas subdimensões relacionadas à orientação, ao atendimento resolutivo e à multidisciplinaridade, mostrando que, para eles, o que torna um serviço conveniente não se resume a uma boa localização e ao horário de funcionamento, embora estes aspectos tenham sido reafirmados como pertinentes a esta dimensão. Por meio do exame dos itens sugeridos, podese afirmar que a subdimensão acesso, incluída pelos participantes significa acesso geográfico.

Importante notar que a disponibilidade dos medicamentos também foi citada como relevante para a conveniência dos serviços. No estudo de Luiza et al. ${ }^{19}$ tanto usuários quanto dispensadores, responsáveis pela dispensação, e prescritores (médicos) consideraram esta dimensão como a mais importante frente às demais. Ao tratar da dispensação de medicamentos, muitas vezes se trata a disponibilidade como um fim em si mesmo. A contribuição dos especialistas mostra que este aspecto impacta também na conveniência - a falta de algum medicamento implicará na necessidade de retorno do usuário em algum outro momento, ou sua busca em algum outro espaço - se houver esforço para manter a integralidade do tratamento. A ausência de algum medicamento no momento em que o usuário necessita pode até não ocasionar na interrupção do tratamento, mas segundo os especialistas tornará a busca pelos esquemas de tratamento pouco conveniente aos usuários.

A dimensão Presença do Medicamento foi entendida como sendo caracterizada por: direito de acesso, disponibilidade, logística, adesão e presença do farmacêutico. Embora tenha recebido uma menor quantidade de itens na primeira rodada do Delfos, esta dimensão manteve a maior parte de seus itens classificadas como sendo pertinentes pelos participantes na segunda rodada do estudo, confirmando a visão mais coesa a respeito desta dimensão. É importante ressaltar que na primeira estrutura (Figura 1) esta dimensão já era composta por itens homogêneos que se referiam à existência do medicamento e em quantidade necessária. A função do medicamento e sua constante necessidade fizeram com que os itens desta dimensão fossem objeto de rápido consenso.

A presença de farmacêuticos e/ou responsáveis pelo provimento do tratamento nas Unidades Dispensadoras de Medicamentos (UDM), entre os especialistas, trouxe a logística como uma subdimensão que compõe a presença do medicamento. O aparecimento deste item fala da complexa cadeia de abastecimento do setor farmacêutico e do relacionamento entre as unidades de saúde e as secretarias municipais, estaduais e o Ministério da Saúde. Este foco ${ }^{20}$, quando investigado mais profundamente no instrumento de trabalho, foi sugerido de forma a explicitar a logística da assistência farmacêutica na esfera estadual. É provável que a ênfase nesta esfera esteja pela sua responsabilidade na provisão de medicamentos para doenças oportunistas, que muitas vezes falha em todos os estados do país.

As subdimensões presença de farmacêutico, logística e 'adesão' aproximam e vinculam o conteúdo da dimensão presença do medicamento à qualidade técnica da dispensação e do medicamento $^{21}$. A presença do medicamento deve ser 
Tabela 1. Itens considerados pertinentes e relevantes pelos especialistas após segunda rodada do Delfos.

Dimensão / Subdimensão

$\begin{array}{cc}\begin{array}{c}\text { Número de } \\ \text { especialistas que } \\ \text { consideraram o } \\ \text { item pertinente }\end{array} & \begin{array}{c}\text { Pontuação de } \\ \text { relevância } \\ \text { do item }\end{array}\end{array}$

Itens

Oportunidade / Conveniência

Profissional que orienta sobre utilização e efeitos dos medicamentos

Atendimento às necessidades individuais no momento da retirada do medicamento

Disponibilidade de horário de atendimento da Unidade Dispensadora de

Medicamentos (UDM)

Acesso ao medicamento

Disponibilidade de medicamentos para infecções oportunistas

Disponibilidade dos ARV

Atendimento Resolutivo

Equipe multidisciplinar para atendimento

Facilidade do acesso a UDM

Disponibilidade / Presença do Medicamento

Direito de acesso ao medicamento necessário

Disponibilidade de todos os medicamentos para o tratamento

Disponibilidade imediata do medicamento de acordo com o prescrito

Fundamental para contribuir com a adesão ao tratamento

Logística do Programa Estadual para abastecer as UDM

Presença do Farmacêutico

Adequação / Qualidade Técnica da Dispensação

Conferência de possíveis dúvidas do usuário

Equipe treinada e capacitada para exercer atividade de dispensação

Orientação ao usuário sobre o uso dos medicamentos contidos na prescrição

Preenchimento adequado dos formulários para dispensação

Profissional Capacitado, treinado, atualizado e que saiba transmitir seu

conhecimento

Recordar a prescrição com o usuário

Capacidade de esclarecer e orientar devidamente os usuários de medicamentos

Confiança do usuário no dispensador

Dispensação conforme a prescrição

Capacidade técnica do profissional que presta as informações na dispensação

Informações recebidas sobre a terapia medicamentosa

Adequação / Qualidade Técnica do Medicamento

Clareza das informações contidas em sua apresentação

Eficiência

Orientação para uso e conservação corretos no período de consumo pelo usuário

Segurança

Facilidade para ser ingerido

Importante para a manutenção da vida da pessoa vivendo com aids

Poucos efeitos adversos (ou "secundários")

Alterações química/física do medicamento

Armazenamento do medicamento

Indicação registrada

Preenchimento de todos os requisitos exigidos em conformidade com a legislação

em vigor

Utilizados para recuperação, manutenção ou prevenção da saúde

Validação pelos órgãos reguladores FDA, EMEA, ANVISA, OMS

Adequação / Ambiência

Local organizado para desenvolvimento da atividade

Local limpo

Garantia de confidencialidade

Garantia de Privacidade

Adequação / Aspectos Interpessoais

Fatores que determinam o grau de confiança entre os usuários e os profissionais

Respeito à cultura, religião, educação, status social, comportamento, vivência

Trabalho em equipe

Satisfação dos usuários com o profissional

Produto das relações estabelecidas entre usuário e profissional de saúde e vice-versa

7

7

13

12

12

6

6

6

6

6
6

7

7

7

6

6

7

7

7

7

7

7

7
7 


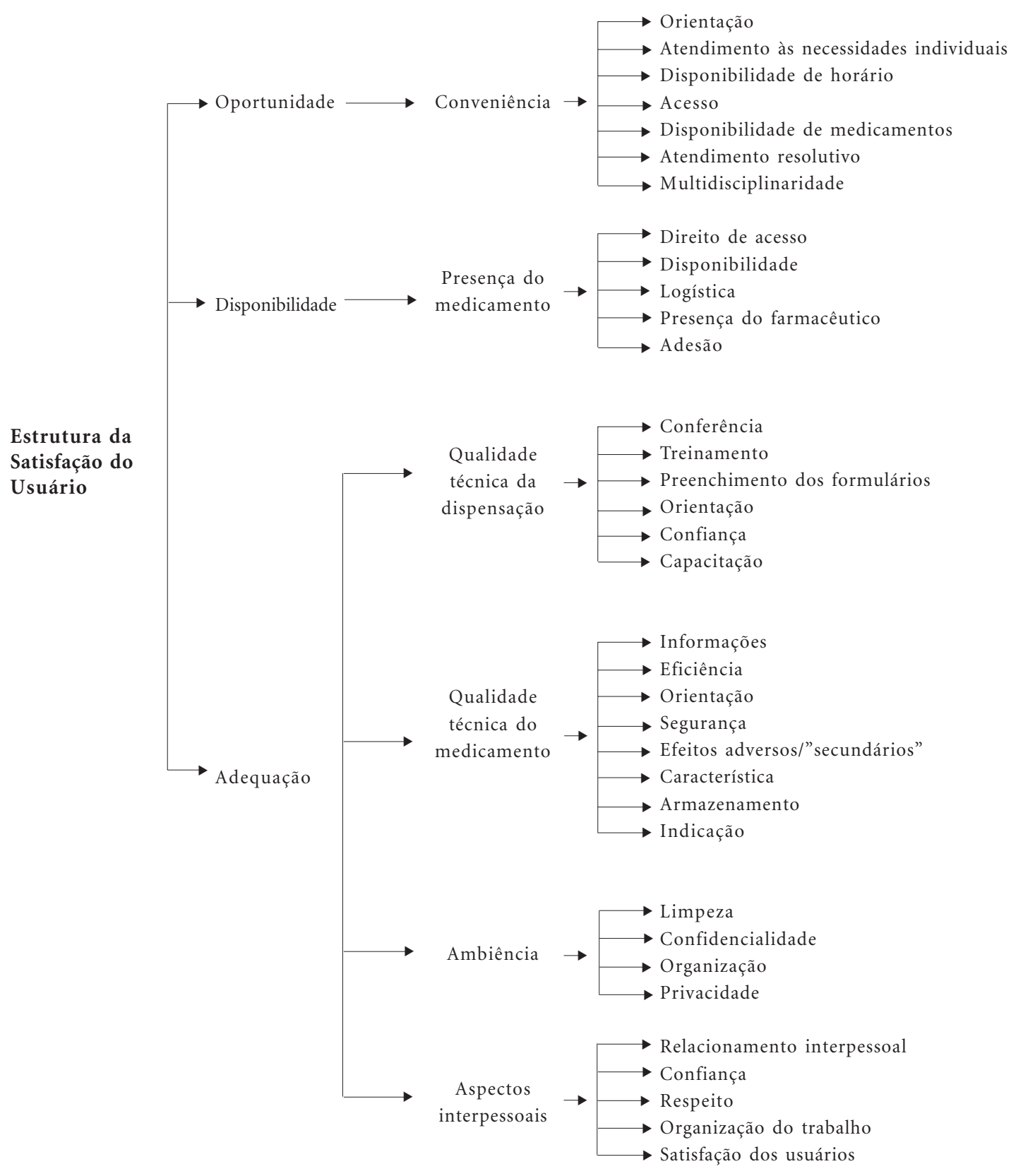

Figura 2. Estrutura da satisfação dos usuários após o delfos

avaliada com foco na disponibilidade e no acesso, fins implícitos desta dimensão, mas também por subdimensões relacionadas às tarefas, aos meios, que devem ser desempenhadas para sua garantia.

A inclusão do farmacêutico como subdimensão resultante do consenso reflete uma preocupação com a legislação. Desde 1973, o Brasil tem legislação que exige a presença do farmacêutico durante todo o horário de funcionamento do estabelecimento $^{22}$. A participação e a troca proporcionada pelo uso de Delfos permitiram que profissionais farmacêuticos integrantes do grupo de especialistas refletissem sobre sua própria contribuição ao desempenho do programa. Ao 
envolver esses profissionais, transparece a importância que atribuem a si mesmos ou ao seu trabalho, ou que consideram que deveriam ter.

Além de múltiplas responsabilidades que envolvem questões legais, técnicas e clínicas, o farmacêutico poderá formar e capacitar outros profissionais para identificar, corrigir ou reduzir possíveis equívocos na terapêutica prescrita. Deve-se considerar que na prática nem todas as unidades dispensadoras contarão integralmente com a presença de profissionais com esta formação específica, mas é necessário garantir a presença de profissionais com atributos que trabalhem para garantir o bom desempenho desta dimensão.

Na Qualidade Técnica da Dispensação, a subdimensão que compunha a primeira estrutura encontra relação próxima com as resultantes do Delfos. O modelo inicial traz como elemento desta dimensão, a acurácia, entendida como verificação da receita, atendimento da quantidade certa e com orientação e os especialistas criaram subdimensões que se bem executadas e avaliadas cumprem o papel de uma dispensação com acurácia, isto é, que envolva capacitação e orientação, existência de profissionais capacitados e orientação adequada com estabelecimento de vínculo de confiança com usuário e registro das atividades estabelecidas.

É possível afirmar que subdimensões como confiança e preenchimento de formulários têm origem na participação de especialistas com experiência na logística interna da dispensação. Os especialistas consideram que na medida em que o processo de trabalho passe por maior organização isto aumentará a qualidade técnica da dispensação.

Ao destacar a confiança e a formação de vínculo com os usuários na orientação oferecida a estes como aspectos relevantes da qualidade técnica da dispensação, as contribuições dos especialistas se aproximam da forma como os usuários julgam esta dimensão. Estudo de Azeredo et al. ${ }^{23}$ apontou que as respostas dos usuários a itens de julgamento da qualidade técnica da dispensação, como a verificação cuidadosa da prescrição, se relacionavam fortemente a atributos da relação interpessoal como a atenção, a confiança e a cordialidade no atendimento.

Para a dimensão Qualidade Técnica do Medicamento, o consenso resultou na inserção de subdimensões como: eficiência, segurança, facilidade de utilização, orientação quanto ao uso e conservação, registro coerente com a indicação, adequação à legislação vigente. A proposta do modelo inicial era mais restrita às características dos medicamentos e sua ação.

Cabe destaque também para o caráter processual da qualidade do medicamento introduzido nesta dimensão pelos especialistas. Neste sentido, a qualidade técnica do medicamento não é assumida a priori, como uma propriedade intrínseca ao produto manufaturado. Antes, deve ser garantida por um engajamento ativo daqueles envolvidos no uso, tanto dispensadores quanto usuários, necessitando de procedimentos de registro e aferição de conformidade legal bem como de orientação para uso e conservação adequados.

No modelo inicial, a dimensão Ambiência era composta por três subdimensões: limpeza, conforto e sinalização. Sob o ponto de vista dos especialistas as subdimensões pactuadas foram: organização do local, limpeza, confidencialidade e privacidade. Estudos anteriores ${ }^{19,23}$ já demonstravam a relação entre aspectos da ambiência ou infraestrutura das unidades dispensadoras de medicamentos ARV com a privacidade e a confidencialidade nos atendimentos. Esses estudos apontaram que a possibilidade de um atendimento com privacidade está fortemente relacionada ao ambiente da dispensação, resultados estes que corroboram a posição dos especialistas ao considerarem estas subdimensões como parte de uma mesma dimensão. Além disso, há correlação entre o ambiente da dispensação, o atendimento com privacidade e o recebimento de orientação sobre o tratamento.

A constituição de uma dimensão de ambiência que inclua aspectos infraestruturais da dispensação, privacidade e confidencialidade do atendimento possibilitará a avaliação de aspectos relacionados à orientação e ao aconselhamento. Segundo Watermeyer ${ }^{24}$, uma comunicação bem sucedida e adequada é necessária para promover a adesão e assegurar que pacientes alcancem os melhores resultados possíveis com o tratamento.

Os especialistas caracterizaram a dimensão Aspectos Interpessoais com elementos relacionados ao grau de confiança entre os usuários e os profissionais da dispensação, ao respeito, à organização do trabalho e à própria satisfação. Se comparada à estrutura inicial, o respeito é a subdimensão que mais aproxima as duas propostas. No entanto, um estudo anterior já apontava que a confiança no trabalho dos dispensadores estava relacionada ao respeito e aos aspectos interpessoais ${ }^{23}$.

Interessante notar a reflexão dos especialistas quanto à organização do trabalho e sua vinculação com o respeito aos usuários. Fica implícito que mais que uma iniciativa de conformidade técnica há uma relação entre organização do trabalho e respeito como aspecto que traz satisfação. Ao avaliar a organização do trabalho, avalia-se o nível de respeito dispensado aos usuários, assim, o respeito se traduz em serviço organizado. 
A subdimensão Orientação foi inserida nas dimensões Conveniência, Qualidade Técnica da Dispensação e Qualidade Técnica do Medicamento por sua importância na assistência farmacêutica e pelas distintas características dos especialistas que fizeram parte do consenso. $\mathrm{O}$ intenso exercício de orientação que considera que pacientes possam sentir desconforto para formular questões ou para externar voluntariamente suas preocupações é uma medida a favor da adesão. A ruptura na comunicação entre profissional de saúde e paciente é apontada como uma dentre as muitas razões que dificultam a compreensão de instruções sobre medicamentos, condição essencial para o sucesso do tratamento ${ }^{24}$.

\section{Considerações Finais}

Este artigo apresentou critérios de julgamento para avaliar a satisfação dos usuários com a dispensação dos medicamentos para o tratamento do HIV/Aids e seu processo de construção por meio de consenso entre diferentes atores sociais. Os critérios, descritos como dimensões e subdimensões, devem ser compreendidos como categorias cognitivas, construídas a partir da teoria de satisfação dos usuários e dispensação de medicamentos e que serão utilizadas no julgamento do programa. Quando aplicados ao foco da avaliação possibilitarão intervenções mais específicas nos diferentes componentes técnicos e estruturais da dispensação.

As subdimensões incorporadas a partir da contribuição dos especialistas, mostram consistência com outros estudos ${ }^{5,19,21,23,24}$, permitiram a ampliação dos domínios das dimensões inicialmente propostas e possibilitaram perceber como são tênues os limites entre elas.

Seguindo os princípios da abordagem participativa nas avaliações, buscou-se a construção coletiva de valores comuns ao utilizar a técnica de Delfos e possibilitar a seleção de profissionais com diferentes inserções na área de medicamentos e assistência farmacêutica, assim como ativistas do movimento social de luta contra Aids com algum tipo de inserção acadêmica. A participação de membros de um GT previamente organizado pelo PN-DST/Aids poderia ser compreendida como um fator limitante das sugestões mas não o foi, seja porque o Grupo já havia encerrado suas tarefas na ocasião deste estudo, seja pela experiência ocupacional dos especialistas ou ainda pela mobilização com o tema. O desafio de expandir a noção de especialistas e reunir pessoas que contribuíssem com sua experiên- cia, representando diferentes partes interessadas foi cumprido e isto pôde ser percebido na troca de informações que possibilitou aos participantes o acesso a novos conteúdos e ao estabelecimento de consenso, construído com base em uma multiplicidade de opiniões e vivências.

A falta de interação direta entre o grupo permitiu a garantia de privacidade e a livre expressão e evitou constrangimentos entre os participantes. No entanto, impossibilitou o aprofundamento da análise de determinadas questões. No modelo construído a partir das contribuições dos especialistas, a satisfação dos usuários foi sugerida como subdimensão de aspectos interpessoais. Aspectos interpessoais é no modelo uma dimensão da estrutura de satisfação dos usuários. Como o objetivo do estudo era estabelecer consenso entre os especialistas, não foram realizadas análises individuais de cada contribuição.

A proposição de estabelecer critérios de avaliação a partir do consenso de um grupo que representasse as diferentes partes interessadas na intervenção buscou contribuir para o desenvolvimento e o fortalecimento de uma estratégia metodológica participativa e ampliar a produção do conhecimento sobre a avaliação de satisfação dos usuários com a dispensação dos medicamentos para o tratamento do HIV/Aids.

\section{Colaboradores}

A Esher participou da pesquisa e desenvolvimento da concepção teórica, elaboração da metodologia, coleta e análise dos dados e redação do texto; E M Santos participou da pesquisa e desenvolvimento da concepção teórica e redação do texto; R Magarinos-Torres e T B Azeredo participaram da elaboração da metodologia, análise dos dados e redação do texto. A revisão final do artigo foi realizada por todos os autores. 


\section{Referências}

1. Alföldi F. Savoir Évaluer En Action Sociale Et Médico-Sociale. Paris: Dunod; 2006.

2. Worthen BR, Sanders JR, Fitzpatrick JL. Avaliação de Programas: concepções e práticas. São Paulo: Editora Gente; 2004.

3. Cousins JB, Whitmore E. Framing participatory evaluation. New Directions for Evaluation 1998; (80): 5-23.

4. Brandão DB, Silva RR, Palos CMC. Da construção de capacidade avaliatória em iniciativas sociais: algumas reflexões. Ensaio: aval. pol. púb. Educ 2005; 13(48):361-374.

5. Esher Â, Santos EM, Azeredo TB, Luiza VL, Osorio-de-Castro CGS, Oliveira MA. Logic Models from an Evaluability Assessment of Pharmaceutical Services for People Living with HIV/AIDS. Cien Saude Colet. No prelo 2010

6. Vaitsman J, Andrade GRB. Satisfação e responsividade: formas de medir a qualidade e a humanização da assistência à saúde. Cien Saude Colet 2005 10(3):599-613.

7. Penchansky DR, Thomas JW. The Concept of Access - Definition and Relationship to Consumer Satisfaction. Med Care 1981; 19(2):127-140.

8. Frenk J, White KL, editors. El Concepto y la Medición de Acessibilidad. In: Frenk J, White KL, editors. Investigaciones sobre servicios de salud. Washington D. C.: Organización Panamericana de la Salud (OPAS); 1992. p. 929-943.

9. Mor V. Developing Indicators of Access to Care: The Case for HIV Disease. In: Access to Health Care in America. Washington: The National Academies Press; 1993.

10. Cunningham WE, Hays RD, Williams KW, Beck KC, Dixon WJ, Shapiro MF. Access to medical care and health-related quality of life for low income persons with symptomatic human immunodeficiency virus. Medical Care 1995; 33(7):739-754.

11. World Health Organization (WHO), Management Sciences for Health (MSH). Defining and Measuring Access To Essential Drugs, Vaccines, and Health Commodities. Ferney-Voltaire: World Health Organization (WHO), Management Sciences for Health (MSH); 2000.

12. Mackeigan LD, Larson LN. Development and Validation of an Instrument to Measure Patient Satisfaction with Pharmacy Services. Medical Care 1989; 27(5):522-536.

13. Ware Jr JE, Snyder MK, Wright WR, Davies A. Defining and Measuring Patient Satisfaction with Medical Care. Evaluation and Program Planning 1983; 6(3-4):247-263.

14. Sitzia J, Wood N. Patient Satisfaction: a Review of Issues and Concepts. Social Science \& Medicine 1997; 45(12):1829-1843.
15. Silva AD. A Framework for Measuring Responsive ness. In: Silva AD.GPE Discussion Paper Series: No. 32. Geneva: World Health Organization (WHO); 1999. p. 42.

16. Epstein S. Impure Science: AIDS, activism, and the politics of knowledge. Berkeley and Los Angeles, California: University of California Press; 1996.

17. Wright JTC, Giovinazzo RA. Delphi - Uma Ferramenta de Apoio ao Planejamento Prospectivo. Cadernos de Pesquisas em Administração 2000; 1(12):5465.

18. Uchoa AC, Gondim GMM, Barreto MA, Rocha NdSPD, Rocha PdM. Utilizando Técnicas de Consenso: potencialidades e limites na avaliação da informação em saúde. In: Hartz Z, Felizberto E, Silva LM, organizadores. Meta-avaliação da Atenção Básica em Saúde: teoria e prática. Rio de Janeiro: Editora Fiocruz; 2008. p. 253-282.

19. Luiza VL, Esher A, Santos EM, Avelar FG, Emmerick ICM, Oliveira MA. Avaliação Nacional da Dispensação de Medicamentos para as PVHA. In: Luiza VL, Esher A, Santos EM, Avelar FG, Emmerick ICM, Oliveira MA. Relatório Final de Pesquisa. Rio de Janeiro: Fundação Oswaldo Cruz(Fiocruz), Escola Nacional de Saúde Pública (ENSP), Núcleo de Assistência Farmacêutica; 2006.

20. Reis AMM, Perini E. Desabastecimento de medicamentos: determinantes, conseqüências e gerenciamento. Cien Saude Colet 2008;13(Supl.):603-610.

21. Vieira FS. Possibilidades de Contribuição do Farmacêutico para a Promoção da Saúde. Cien Saude Colet 2007; 12(1):213-220.

22. Brasil. Congresso Nacional. Lei N. 5991, de 17 de dezembro de 1973. Dispõe sobre o Controle Sanitário do Comércio de Drogas, Medicamentos, Insumos Farmacêuticos e Correlatos, e dá outras Providências. Diário Oficial da União 1973; $18 \mathrm{dez}$.

23. Azeredo TB, Oliveira MA, Luiza VL, Esher Â, Campos MR. User satisfaction with pharmacy services in the Brazilian National STD/AIDS Program: validity and reliability issues. Cad Saude Publica 2009; 25(7):1597-1609.

24. Watermeyer PCJ. "Tell me so I know you understand": pharmacists verification of patients comprehension of antiretroviral dosage instructions in a cross-cultural context. Patient Educ Couns 2009; 75(2):205-213.

Artigo apresentado em 17/08/2010

Aprovado em 29/12/2010

Versão final apresentada em 10/01/2011 